\title{
Osteitis and synovitis, but not bone erosion, is associated with proteoglycan loss and microstructure damage in the cartilage of patients with rheumatoid arthritis
}

\author{
Barbara Herz, ${ }^{1}$ Andreas Albrecht, ${ }^{1}$ Matthias Englbrecht, ${ }_{1}^{1}$ Götz H Welsch, ${ }^{2}$ \\ Michael Uder, ${ }^{3}$ Nina Renner, ${ }^{2}$ Philipp Schlechtweg, ${ }^{3}$ Dominik Paul, ${ }_{1}^{4}$ Lars Lauer, ${ }^{4}$ \\ Klaus Engelke, ${ }^{5}$ Rolf Janka, ${ }^{3}$ Jürgen Rech, ${ }^{1}$ Georg Schett, ${ }^{1}$ Stephanie Finzel ${ }^{1}$
}

Handling editor Tore K Kvien

- Additional material is published online only. To view please visit the journal online (http://dx.doi.org/10.1136/ annrheumdis-2012-202850)

${ }^{1}$ Department of Internal Medicine 3, Rheumatology and Immunology, University of Erlangen-Nuremberg, Erlangen, Germany

${ }^{2}$ Department of Traumatic Surgery, University of ErlangenNuremberg, Erlangen, Germany ${ }^{3}$ Institute of Radiology, University of ErlangenNuremberg, Erlangen, Germany ${ }^{4}$ Siemens Healthcare, Erlangen, Germany

${ }^{5}$ Institue of Medical Physics, University of ErlangenNuremberg, Erlangen, Germany

\section{Correspondence to} Dr Stephanie Finzel, Department of Internal Medicine 3, Rheumatology and Immunology; University of Erlangen-Nuremberg; Krankenhausstrasse 12 Erlangen D-91054; Germany; stephanie.finzel@uk-erlangen. de

Accepted 1 April 2013 Published Online First 26 April 2013

\footnotetext{
To cite: Herz $B$, Albrecht $A$, Englbrecht $\mathrm{M}$, et al. Ann Rheum Dis 2014;73: 1101-1106.
}

\section{ABSTRACT}

Objectives To investigate the relation between anatomic changes of the synovium, the bone, the bone marrow and the cartilage to biochemical properties of the cartilage in patients with rheumatoid arthritis (RA). Methods 33 patients with RA received 3-T MRI scans of the metacarpophalangeal joints. Two independent methods, (A) the delayed gadolinium enhanced MRI of the cartilage (dGEMRIC, T2-mapping), which was used to assess the biochemical properties of the cartilage; (B) synovitis, osteitis and bone erosions were quantified according to the RA MRI scoring (RAMRIS) method and cartilage thickness (CT), interbone joint space (IBJS, distance between proximal and distal bone surface) and intercartilage joint space (ICJS, distance between proximal and distal cartilage surface) were measured. Results Biochemical changes of the cartilage, corresponding to low dGEMRIC and high T2 values, were more likely to be seen in joints with decreased IBJS and ICJS as well as decreased CT. For instance, dGEMRIC was directly correlated to the IBJS ( $p=0.001)$ and ICJS ( $p=0.001)$, whereas T2 mapping was inversely correlated to IBJS and ICJS (both $p=0.017$ ). Moreover, the degree of osteitis, and to some extent synovitis, was correlated to biochemical cartilage changes as measured by dGEMRIC ( $p=0.003)$ or the T2 mapping $(p=0.013)$. By contrast, bone erosions did not correlate to the degree of biochemical cartilage changes.

Discussion These data support the concept that synovitis and osteitis may be two main triggers for cartilage damage. Thus, the actual inflammatory state of a joint, but not so much the degree of bone erosion, appears to influence cartilage properties in RA.

\section{INTRODUCTION}

The clinical picture of joint involvement in rheumatoid arthritis (RA) is a composite of inflammation, bone erosion and cartilage destruction. Inflammation of the synovial membrane (synovitis) as well as the juxta-articular bone marrow (osteitis) is considered to drive the destruction of bone and cartilage leading to bone erosion and thinning of the cartilage layer. Whereas, instruments have been developed to assess inflammation and bone erosion in patients with RA, the assessment of cartilage destruction in RA is still challenging. Thus, inflammation can easily be assessed by clinical examination (joint swelling) as well as by established imaging techniques such as high-resolution ultrasound and conventional MRI. Also, bone erosion can be well documented by conventional radiography. By contrast, there are very limited data on directly visualising cartilage and cartilage damage in RA. Nonetheless, instruments have been developed to indirectly measure signs of morphological cartilage changes in patients with RA. Thus, narrowing of the radiographic joint space has been recognised as a consistent finding in patients with RA since many years. ${ }^{12}$ Also, documentation of the change of joint space narrowing over time is widely used to document the progression of structural damage to the cartilage in RA. ${ }^{34}$

Direct assessment of cartilage changes in RA, however, appears to be of fundamental importance, since morphological changes in the cartilage have been shown to be related to decreased physical function in RA. ${ }^{5}{ }^{6}$ Moreover, microscopic changes of the cartilage, that is, biochemical changes and alterations in collagen network and water content, may long precede the morphological changes of the cartilage and might, therefore, be a more sensitive and accurate method to characterise the functional properties of cartilage in RA. ${ }^{7}$ Detection of such submorphological changes may require improving of imaging tools to directly assess the articular cartilage of the small finger joints in patients with RA. However, there is a substantial challenge in applying established methods to measure cartilage proteoglycan content and the alignment of the collagen fibres in the small joints based on the dimensions of the cartilage in small finger joints with a width of less than $1 \mathrm{~mm}$.

Recently, thanks to improvements in MRI technology, techniques to assess the biochemical properties of cartilage could be applied in smaller joints in humans, which have been previously validated in larger joints, that is, the knee joint. ${ }^{8-14}$ These techniques make use of the fact that cartilage damage leads to proteoglycan loss and a change in the collagen fibre network before loss of cartilage structure becomes apparent. Specific MRI sequences, such as delayed gadolinium-enhanced MRI of the cartilage (dGEMRIC) have been used to quantify proteoglycan loss, ${ }^{8-12}$ whereas T2 mapping has been developed to measure changes in water content and collagen network in the cartilage. ${ }^{13} 14$ 
So far, a few studies have applied dGEMRIC and T2 mapping for the assessment of cartilage changes in healthy control subjects and patients with RA. ${ }^{7} 111516$ These data, conducted in a small number of subjects, have shown that both techniques are feasible to measure the biochemical properties of cartilage in the finger joints. In addition, these studies revealed that patients with RA appear to lose cartilage proteoglycan in contrast with healthy controls. ${ }^{7} 1415$ This loss obviously occurs early in the course of the disease, and it seems that proteoglycan loss precedes the morphological changes in the cartilage. However, so far, it is unclear how these changes are related to the macroscopic morphological properties of cartilage as well as the joint space width. In addition, it is still elusive, how validated imaging instruments to assess joint inflammation and bone erosion in RA, such as the RA MRI scoring (RAMRIS) synovitis, osteitis and bone erosion scores ${ }^{17} 18$ are related to the biochemical properties of the cartilage.

Hypothesising a close relation between joint inflammation and bone erosions on the one hand and the biochemical alterations of the cartilage on the other hand, we conducted a highresolution MRI study in patients with established RA aiming to compare the extent of synovitis, osteitis and bone erosions with cartilage microstructure assessed by dGEMRIC and T2 mapping.

\section{METHODS}

\section{Patients' characteristics}

A total number of 33 patients from the Rheumatology Outpatient Clinic of the University Clinic of Erlangen were included in this study, all of them fulfilling the new ACR/ EULAR classification criteria for RA. ${ }^{19}$ In three patients, the metacarpophalangeal (MCP) joints showed very severe destruction making analysis of the articular cartilage impossible; one patient could not be assessed due to claustrophobia.

Demographic parameters, such as gender, age and disease duration, as well as clinical parameters, such as C-reactive protein (CRP) and erythrocyte sedimentation rate (ESR) levels, disease activity score (DAS) 28 , rheumatoid factor and anticitrullinated protein antibodies were assessed at the day of the MRI investigation by a rheumatologist blinded to the MRI findings. Furthermore, medical treatment with glucocorticoids, DMARDs and biologicals was recorded.

\section{Image acquisition}

Each patient received a 3-T MRI-scan of the dominantly affected hand (Verio, Siemens Healthcare, Erlangen, Germany) with two $4 \mathrm{~cm}$ loop surface coils, designed for high-resolution imaging of the cartilage $(0.26 \times 0.26 \times 1.5 \mathrm{~mm}$ voxel size $)$, covering the volar and dorsal side of the second and third MCP joints. Patients had to lie down in a prone position with the hand being fixed with the thumb up in a brace in order to avoid movement artefacts. The MR protocol contained morphological sequences to control positioning of biochemical sequences. For T2 mapping, a multiecho, spin-echo (SE) sequence with six echoes was used; visualisation of cartilage was done by $3 \mathrm{D}$ dual-flip-angle gradient-echo (GRE) technique for T1dGEMRIC (figure 1A,B). The maps showing the $\mathrm{T} 2$ and $\mathrm{T} 1$ relaxation times were calculated by software provided by the manufacturer of the MRI scanner (Maplt, Siemens-Healthcare, Erlangen, Germany). $0.2 \mathrm{mmol} / \mathrm{kg}$ Gd(DTPA) ${ }^{-}$(Magnevist, Schering, Berlin, Germany) was administered intravenously $30 \mathrm{~min}$ before the quantitative T1dGEMRIC mapping, and patients had to move their hand for $5 \mathrm{~min}$ after the administration to ensure adequate distribution of contrast agent. The repetition time was
$15 \mathrm{~ms}$ in dGEMRIC and $1200 \mathrm{~ms}$ in T2 mapping. The echo time was $3.83 \mathrm{~ms}$ in dGEMRIC and 15.8, 314.6, 47.4, 63.2, 79.0 and $94.8 \mathrm{~ms}$ in T2 mapping. The flip angle was $5^{\circ}$ and $26^{\circ}$ in dGEMRIC and $180^{\circ}$ in T2 mapping. The field of view was $100 \times 100 \mathrm{~mm}$, the pixel matrix $384 \times 384$, and the slice thickness $1.5 \mathrm{~mm}$; thereby, these parameters were the same in the two sequences. The number of slides was 32 in dGEMRIC and 10 in T2 mapping. The voxel size was $0.26 \times 0.26 \times 1.5 \mathrm{~mm}$, the interpolated voxel size was $0.13 \times 0.13 \times 1.5 \mathrm{~mm}$, and the bandwidth $200 \mathrm{~Hz} / \mathrm{Px}$ both in dGEMRIC and T2 mapping.

\section{Image analysis}

MCP heads and bases were scored semiquantitatively for synovitis, osteitis and bone erosion in the morphological sequences using the RAMRIS system (Leonardo Workstation, SiemensHealthcare, Erlangen, Germany). ${ }^{17}{ }^{18}$ Interbone joint space (IBJS), defined as the distance between the proximal and the distal bone surface, intercartilage joint space (ICJS), as the plain space between the proximal and distal cartilage layer, proximal (CTprox) and distal cartilage thickness (CTdist) and total cartilage thickness (TCT), as the sum of the two cartilage layers, were measured perpendicular to the subchondral bone in the ulnar, medial und radial region of the joint (figure 1).

A pixel-wise, monoexponential, non-negative least-squares-fit analysis was used for the calculation of the T2 maps, and the T1dGEMRIC maps were obtained based on the analysis of the two flip-angles as proposed by Trattnig et al. ${ }^{10} \mathrm{Six}$ regions of interest (ROI), three proximal and three distal, covering the whole cartilage layers and leaving out the joint space, were drawn manually into the morphological slice nearest to the middle of MCP joints 2 and 3, respectively. Those regions were than copied into the slice showing the same position in the biochemical T2 and T1 maps to record the relaxation times. All analyses were done by two independent readers for interobserver reliability, one of them scoring twice to additionally test for intraobserver reliability. Both of them were blinded to clinical findings and treatment of the patients.

\section{Statistical analysis}

The recorded values were analysed for correlation using nonparametric Spearman's rho. We tested for correlation between age, DAS28, disease duration, CRP and ESR. Means for dGEMRIC and T2 in the metacarpal head and the phalangeal base separately and for the whole MCP 2 and 3 joints were calculated. dGEMRIC and T2 means for the whole joints were tested for correlation with clinical parameters, and means for head and base were tested separately with values for synovitis, bone erosions and osteitis. Furthermore, dGEMRIC and T2 values of the six ROIs in each joint were analysed for correlation with the values of IBJS, ICJS, TCT and CT proximal and distal at the same position in the joint. After that, we performed a Bonferroni-Holm adjustment for multiple testing per characteristic, leading to the following critical $\mathrm{p}$ values that were applied from strongest to weakest. Spearman's correlation per line, and rounded to three digits after the decimal point $\left(\mathrm{p}_{1}=0.013 ; \mathrm{p}_{2}=0.017 ; \mathrm{p}_{3}=0.025 ; \mathrm{p}_{4}=0.05\right)$. If one coefficient failed to reach significance according to these critical $p$ values, all subsequent correlation coefficients of the same characteristic were declared non-significant. Intraobserver reliability was calculated using a Spearman's correlation. Interobserver reliability was computed via Intra Class Coefficients (ICCs). IBM SPSS statistics 19.0 (SPSS-Institute, Chicago, USA) was used, and a $\mathrm{p}$ value of less than 0.05 was considered statistically significant. 
A

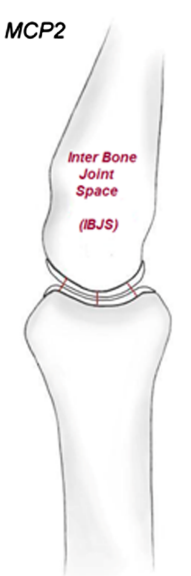

$\mathrm{B}$

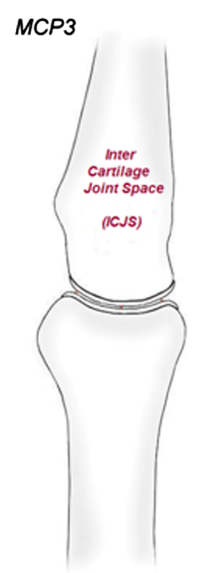

B

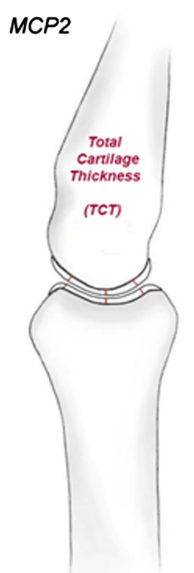

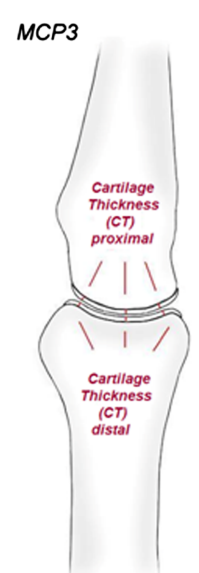
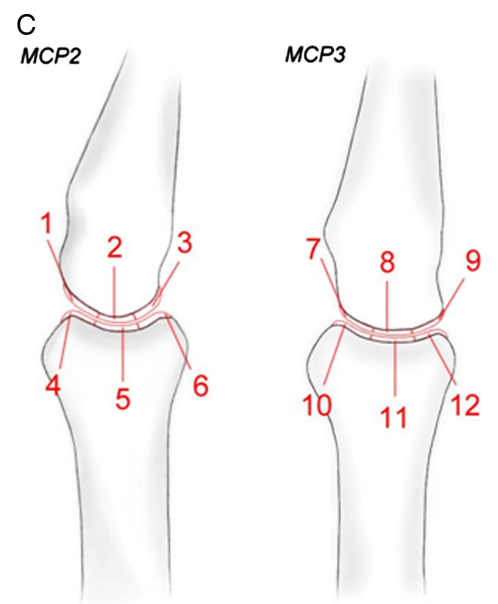

Figure 1 Methodology of assessment of cartilage parameters. Schematic drawing of the metacarpophalangeal (MCP) joints 2 and 3 . (A) Evaluation of interbone joint space (left, IBJS), and intercartilage joint space (right, ICJS). (B) Evaluation of proximal, distal and TCT. (C) Region of interest (ROI) evaluation for T2 mapping and delayed gadolinium-enhanced MRI of the cartilage (dGEMRIC) is shown. Six regions-of-interest, three in the metacarpal head, three in the phalangeal base, are depicted in each joint.

\section{RESULTS}

\section{Clinical and MR imaging characteristics}

A complete set of cartilage parameters was performed in 29 patients, 22 of them were women, and seven of them men. Mean $\pm S D$ age was $49.7 \pm 13.3$ years, mean \pm SD disease duration was $7.7 \pm 6.7$ years with a mean \pm SD DAS28 of $3.2 \pm 1.5$. $52 \%$ of the patients showed rheumatoid factor positivity and positive autoantibodies against citrullinated proteins (ACPA) were also found in $52 \%$ of the patients; $48 \%$ of the patients used biological therapy (8 TNFi, 4 tocilizumab, 2 rituximab), $69 \%$ methotrexate and 52\% glucocorticoids. All the aforementioned 29 patients were scored according to the RAMRIS method. A summary of the quantification of the standard RAMRIS results are shown in table 1.

\section{Assessment of ICJS and IBJS}

We next assessed the dimensions of the articular cartilage in our patients by measuring the ICJS and the IBJS as well as the width of the proximal and distal cartilage layer as detailed in figure

\begin{tabular}{llll} 
Table 1 & RAMRIS-scores & & \\
\hline Joint & Compartment & Mean \pm SD & Median (minimum; maximum) \\
\hline Synovitis & & & \\
MCP II & Total & $1.39 \pm 0.86$ & $1.17(0.00 ; 3.50)$ \\
MCP III & Total & $1.30 \pm 0.82$ & $1.00(0.00 ; 3.00)$ \\
Erosion & & \\
MCP II & MCH & $0.91 \pm 1.49$ & $0.00(0.00 ; 6.00)$ \\
MCP II & PB & $0.99 \pm 1.56$ & $0.00(0.00 ; 6.00)$ \\
MCP III & MCH & $0.36 \pm 0.61$ & $0.00(0.00 ; 3.00)$ \\
MCP III & PB & $0.14 \pm 0.35$ & $0.00(0.00 ; 1.00)$ \\
Osteitis & & & \\
MCP II & MCH & $0.18 \pm 0.47$ & $0.00(0.00 ; 2.00)$ \\
MCP II & PB & $0.28 \pm 0.65$ & $0.00(0.00 ; 3.00)$ \\
MCP III & MCH & $0.07 \pm 0.26$ & $0.00(0.00 ; 1.00)$ \\
MCP III & PB & $0.06 \pm 0.22$ & $0.00(0.00 ; 1.00)$ \\
\hline Values for RAMRIS synovitis, erosion and osteitis scores. \\
n=29. \\
MCP, metacarpophalangeal joint; MCH, metacarpal head; PB, phalangeal base.
\end{tabular}

1A,B. When measuring the ICJS, very consistent results were found in all regions of MCP2 and MCP3 joints. The IBJS was approximately threefold wider than the ICJS in all six regions of the MCP 2 and 3 joints assessed in this study. Consistent results were found among the different regions of the MCP2 and MCP3 joints. Results are shown in detail in table 2.

\section{Assessment of CT}

We next aimed to directly evaluate the cartilage width by measuring the distance between the cartilage-bone interphase and the cartilage surface in all aforementioned regions (figure 1B). Width of the articular cartilage was similar among the phalangeal bases and the metacarpal heads. Also, very consistent results were found when comparing the MCP2 with the MCP3 joint, showing only a minimally thinner cartilage in the MCP3 than MCP2 joint. Details of the results of the individual joint regions are outlined in table 2 .

\section{Assessment of cartilage microstructure by dGEMRIC and T2 mapping}

Analysis of dGEMRIC maps allow to assess the proteoglycan content of the articular cartilage, as detailed in figure $2 \mathrm{~B}$. dGEMRIC values were significantly higher, indicating higher proteoglycan content in the phalangeal bases than the metacarpal heads. T2 mapping, which assesses water content and collagen microstructure of the cartilage, was also used to analyse cartilage microstructure. Analysis of T2 mapping is shown in figure 2C. High values indicate increased water content and damaged collagen fibre network in cartilage. Again, the phalangeal bases were better preserved than in the metacarpal heads. Details of results are shown in table 3 . Intraobserver reliability for dGEMRIC was $0.64-0.88$ and $0.73-0.97$ for T2 mapping. Interobserver reliability for dGEMRIC was $0.44-0.85$ and 0.30-0.96 for T2 mapping.

\section{Correlation of microstructural and macrostructural changes in the cartilage}

Having shown that measurement of cartilage microstructure is feasible in the MCP joints and reveals consistent results by two different techniques we were interested in how these measures relate to cartilage and joint space width. dGEMRIC results were 
Table 2 Interbone joint space (IBJS), intercartilage joint space (ICJS) and cartilage thickness (CT)

\begin{tabular}{llll}
\hline Joint & Compartment & Mean \pm SD & Median (minimum; maximum) \\
\hline IBJS & & & \\
MCP II & Radial & $1.87 \pm 0.44$ & $1.73(1.20 ; 3.00)$ \\
MCP II & Medial & $1.74 \pm 0.46$ & $1.87(1.50 ; 2.60)$ \\
MCP II & Ulnar & $1.82 \pm 0.44$ & $1.93(1.10 ; 2.60)$ \\
MCP III & Radial & $1.63 \pm 0.40$ & $1.60(0.90 ; 2.50)$ \\
MCP III & Medial & $1.51 \pm 0.47$ & $1.50(0.60 ; 2.60)$ \\
MCP III & Ulnar & $1.58 \pm 0.40$ & $1.53(0.80 ; 2.60)$ \\
ICJS & & & \\
MCP II & Radial & $0.67 \pm 0.23$ & $0.57(0.40 ; 1.40)$ \\
MCP II & Medial & $0.58 \pm 0.23$ & $0.57(0.10 ; 1.20)$ \\
MCP II & Ulnar & $0.65 \pm 0.25$ & $0.67(0.30 ; 1.30)$ \\
MCP III & Radial & $0.54 \pm 0.18$ & $0.53(0.20 ; 1.00)$ \\
MCP III & Medial & $0.47 \pm 0.20$ & $0.43(0.10 ; 1.00)$ \\
MCP III & Ulnar & $0.53 \pm 0.19$ & $0.50(0.20 ; 1.00)$ \\
Proximal CT (Metacarpal head) & & \\
MCP II & Radial & $0.58 \pm 0.12$ & $0.60(0.30 ; 0.80)$ \\
MCP II & Medial & $0.52 \pm 0.12$ & $0.53(0.10 ; 0.70)$ \\
MCP II & Ulnar & $0.60 \pm 0.13$ & $0.60(0.30 ; 0.80)$ \\
MCP III & Radial & $0.53 \pm 0.13$ & $0.53(0.30 ; 0.80)$ \\
MCP III & Medial & $0.51 \pm 0.16$ & $0.50(0.20 ; 0.90)$ \\
MCP III & Ulnar & $0.58 \pm 0.15$ & $0.60(0.30 ; 0.80)$ \\
Distal CT (Phalangeal base) & & \\
MCP II & Radial & $0.62 \pm 0.16$ & $0.60(0.40 ; 1.00)$ \\
MCP II & Medial & $0.63 \pm 0.16$ & $0.63(0.20 ; 1.30)$ \\
MCP II & Ulnar & $0.60 \pm 0.13$ & $0.57(0.40 ; 0.90)$ \\
MCP III & Radial & $0.56 \pm 0.20$ & $0.53(0.30 ; 1.40)$ \\
MCP III & Medial & $0.54 \pm 0.15$ & $0.57(0.30 ; 0.90)$ \\
MCP III & Ulnar & $0.52 \pm 0.11$ & $0.30(0.30 ; 0.70)$ \\
Total CT & & & \\
MCP II & Radial & $1.20 \pm 0.24$ & $1.13(0.70 ; 1.80)$ \\
MCP II & Medial & $1.16 \pm 0.26$ & $1.20(0.40 ; 1.60)$ \\
MCP II & Ulnar & $1.20 \pm 0.24$ & $1.23(0.80 ; 1.60)$ \\
MCP III & Radial & $1.09 \pm 0.28$ & $1.07(0.70 ; 1.80)$ \\
MCP III & Medial & $1.04 \pm 0.29$ & $1.00(0.50 ; 1.60)$ \\
MCP III & Ulnar & $1.05 \pm 0.23$ & $1.10(0.60 ; 1.60)$ \\
\hline Values for interbone and ICJS and for proximal, distal and TCT. \\
n=29. & & \\
MCP, metacarpophalangeal joint. & & \\
& & & \\
& &
\end{tabular}

\begin{tabular}{llll} 
Table 3 & dGEMRIC and T2 scores & \\
\hline Joint & Compartment & Mean \pm SD & Median (minimum; maximum) \\
\hline dGEMRIC & & \\
MCP II & MCH & $642 \pm 222$ & $603(191 ; 1260)$ \\
MCP II & PB & $937 \pm 325$ & $949(284 ; 1723)$ \\
MCP III & MCH & $631 \pm 185$ & $604(297 ; 1046)$ \\
MCP III & PB & $949 \pm 363$ & $908(306 ; 2250)$ \\
T2 Mapping & & & \\
MCP II & MCH & $39.1 \pm 22.1$ & $30.5(13.6 ; 105.4)$ \\
MCP II & PB & $34.3 \pm 21.3$ & $27.6(13.9 ; 114.6)$ \\
MCP III & MCH & $38.6 \pm 20.1$ & $34.5(14.7 ; 109.4)$ \\
MCP III & PB & $30.8 \pm 15.3$ & $27.0(14.4 ; 71.2)$ \\
\hline Values for dGEMRIC and T2 mapping. & \\
$n=29$. &
\end{tabular}

significantly correlated with the IBJS and with CT even after correction for multiple testing (table 4 and online supplementary table). Thus, low dGEMRIC values were associated with joint space narrowing as well as decreased cartilage width. Very similar results were found for T2 mapping, which showed, that higher values in T2 were related to lower CT and smaller joint space suggesting that the biochemical changes in cartilage microstructure are closely associated with the morphological changes of the articular cartilage.

\section{Correlation of RAMRIS synovitis, osteitis and bone erosion with cartilage microstructural changes}

Based on the complete quantitative assessment of inflammation, bone erosion, as well as cartilage macrostructural and microstructural changes in our cohort of patients with RA, we were interested to determine the process, which is most tightly associated with cartilage damage. We therefore correlated the results of RAMRIS-based quantification of synovitis, osteitis and bone erosion to cartilage microstructural parameters. Interestingly, the degree of osteitis as well as synovitis was most tightly correlated with low dGEMRIC and high T2 values indicative for cartilage microstructure damage. Significant correlations between low dGEMRIC values and the degree of synovitis were found in the MCP3 joints, whereas correlations were less pronounced in the
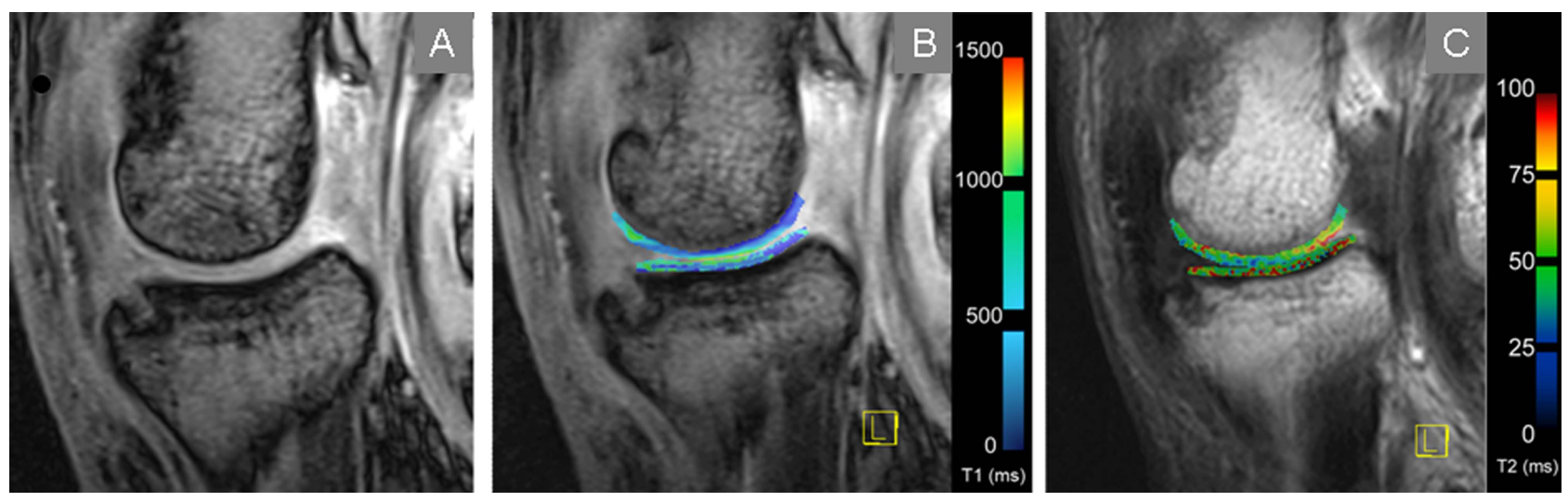

Figure 2 Examples of MRI of the cartilage of the MCP joints. (A) Morphological T1 image of the metacarpophalangeal (MCP) joint 2 with an erosion. (B) Corresponding delayed gadolinium-enhanced MRI of the cartilage (dGEMRIC) map and (C) corresponding T2 map. 
Table 4 Correlation between synovits, osteitis and bone erosion and cartilage parameters to biochemical cartilage properties

\begin{tabular}{|c|c|c|c|c|}
\hline & $\begin{array}{l}\text { MCP2/ } \\
\text { MCH }\end{array}$ & MCP2/PB & MCP3/MCH & MCP3/PB \\
\hline \multicolumn{5}{|l|}{ dGEMRIC } \\
\hline Cartilage thickness & $\begin{array}{l}r_{s}=0.364 \\
p=0.052\end{array}$ & $\begin{array}{l}r_{s}=0.197 \\
p=0.350\end{array}$ & $\begin{array}{l}r_{s}=0.399^{*} \\
p=0.032^{*}\end{array}$ & $\begin{array}{l}r_{s}=0.435^{*} \\
p=0.018^{*}\end{array}$ \\
\hline $\begin{array}{l}\text { Total cartilage } \\
\text { thickness }\end{array}$ & $\begin{array}{l}r_{s}=0.177 \\
p=0.359\end{array}$ & $\begin{array}{l}r_{s}=0.220 \\
p=0.252\end{array}$ & $\begin{array}{l}r_{s}=0.429^{*} \\
p=0.020^{*}\end{array}$ & $\begin{array}{l}r_{s}=0.486^{* *} \\
p=0.008^{* *}\end{array}$ \\
\hline $\begin{array}{l}\text { Interbone joint } \\
\text { space }\end{array}$ & $\begin{array}{l}r_{s}=0.370^{*} \\
p=0.048^{*}\end{array}$ & $\begin{array}{l}r_{s}=0.331 \\
p=0.079\end{array}$ & $\begin{array}{l}r_{s}=0.392^{*} \\
p=0.035^{*}\end{array}$ & $\begin{array}{l}r_{s}=0.572^{* *} \\
p=0.001^{* *}\end{array}$ \\
\hline $\begin{array}{l}\text { Intercartilage joint } \\
\text { space }\end{array}$ & $\begin{array}{l}\mathrm{r}_{\mathrm{s}}=0.488^{* *} \\
\mathrm{p}=0.007^{* *}\end{array}$ & $\begin{array}{l}\mathrm{r}_{\mathrm{s}}=0.590^{* *} \\
\mathrm{p}=0.001^{* *}\end{array}$ & $\begin{array}{l}\mathrm{r}_{\mathrm{s}}=0.261 \\
\mathrm{p}=0.171\end{array}$ & $\begin{array}{l}r_{s}=0.475^{* *} \\
p=0.009^{* *}\end{array}$ \\
\hline RAMRIS synovitis & $\begin{array}{l}r_{s}=-0.150 \\
p=0.438\end{array}$ & $\begin{array}{l}r_{s}=-0.190 \\
p=0.323\end{array}$ & $\begin{array}{l}\mathrm{r}_{\mathrm{s}}= \\
-0.535^{* *} \\
\mathrm{p}=0.003^{* *}\end{array}$ & $\begin{array}{l}r_{s}=-0.323 \\
p=0.087\end{array}$ \\
\hline RAMRIS osteitis & $\begin{array}{l}r_{s}=-0.092 \\
p=0.634\end{array}$ & $\begin{array}{l}r_{s}=-0.259 \\
p=0.174\end{array}$ & $\begin{array}{l}r_{s}=-0.130 \\
p=0.501\end{array}$ & $\begin{array}{l}r_{s}= \\
-0.407^{*} \\
p=0.029^{*}\end{array}$ \\
\hline RAMRIS erosion & $\begin{array}{l}r_{s}=-0.163 \\
p=0.397\end{array}$ & $\begin{array}{l}r_{s}=-0.134 \\
p=0.489\end{array}$ & $\begin{array}{l}r_{s}=-0.259 \\
p=0.174\end{array}$ & $\begin{array}{l}r_{s}=-0.203 \\
p=0.290\end{array}$ \\
\hline \multicolumn{5}{|l|}{ T2 Mapping } \\
\hline Cartilage thickness & $\begin{array}{l}r_{s}=-0.407^{*} \\
p=0.031^{*}\end{array}$ & $\begin{array}{l}r_{s}=-0.318 \\
p=0.099\end{array}$ & $\begin{array}{l}r_{s}=-0.298 \\
p=0.116\end{array}$ & $\begin{array}{l}r_{s}= \\
-0.383^{*} \\
p=0.040^{*}\end{array}$ \\
\hline $\begin{array}{l}\text { Total cartilage } \\
\text { thickness }\end{array}$ & $\begin{array}{l}r_{s}=-0.313 \\
p=0.105\end{array}$ & $\begin{array}{l}r_{s}=-0.279 \\
p=0.150\end{array}$ & $\begin{array}{l}r_{s}=-0.374^{*} \\
p=0.046^{*}\end{array}$ & $\begin{array}{l}r_{s}=-0.345 \\
p=0.067\end{array}$ \\
\hline $\begin{array}{l}\text { Interbone joint } \\
\text { space }\end{array}$ & $\begin{array}{l}r_{s}=-0.262 \\
p=0.178\end{array}$ & $\begin{array}{l}r_{s}=-0.239 \\
p=0.221\end{array}$ & $\begin{array}{l}r_{s}=-0.439^{*} \\
p=0.017^{*}\end{array}$ & $\begin{array}{l}\mathrm{r}_{\mathrm{s}}= \\
-0.381^{*} \\
\mathrm{p}=0.041^{*}\end{array}$ \\
\hline $\begin{array}{l}\text { Intercartilage joint } \\
\text { space }\end{array}$ & $\begin{array}{l}r_{s}=-0.205 \\
p=0.295\end{array}$ & $\begin{array}{l}r_{s}=-0.308 \\
p=0.111\end{array}$ & $\begin{array}{l}\mathrm{r}_{\mathrm{s}}=-0.441^{*} \\
\mathrm{p}=0.017^{*}\end{array}$ & $\begin{array}{l}r_{s}= \\
-0.405^{*} \\
p=0.029^{*}\end{array}$ \\
\hline RAMRIS synovitis & $\begin{array}{l}r_{s}=0.122 \\
p=0.536\end{array}$ & $\begin{array}{l}r_{s}=0.120 \\
p=0.542\end{array}$ & $\begin{array}{l}r_{s}=0.333 \\
p=0.077\end{array}$ & $\begin{array}{l}r_{s}=0.446^{*} \\
p=0.015^{*}\end{array}$ \\
\hline RAMRIS osteitis & $\begin{array}{l}r_{s}=0.271 \\
p=0.163\end{array}$ & $\begin{array}{l}r_{s}=0.464^{* *} \\
p=0.013^{* *}\end{array}$ & $\begin{array}{l}r_{s}=0.374^{*} \\
p=0.046^{*}\end{array}$ & $\begin{array}{l}r_{s}=0.374^{*} \\
p=0.046^{*}\end{array}$ \\
\hline RAMRIS erosion & $\begin{array}{l}r_{s}=0.126 \\
p=0.524\end{array}$ & $\begin{array}{l}r_{s}=0.292 \\
p=0.132\end{array}$ & $\begin{array}{l}r_{s}=0.303 \\
p=0.110\end{array}$ & $\begin{array}{l}r_{s}=0.413^{*} \\
p=0.026^{*}\end{array}$ \\
\hline
\end{tabular}

Correlation analysis of joint space values with dGEMRIC and T2 Mapping. Spearman's correlation coefficient $\left(r_{s}\right)$ and $p$ values $(n=29)$. For each characteristic (ie, four tests per line) Bonferroni-Holm adjustment for multiple testing was applied, leading to the following critical $p$ values that were applied from strongest to weakest. Spearman's correlation per line and rounded to three digits after the decimal point $\left(p_{1}=0.013\right.$; $\left.p_{2}=0.017 ; p_{3}=0.025 ; p_{4}=0.05\right)$. If one coefficient failed to reach significance according to these critical $p$ values, all subsequent correlation coefficients of the same characteristic were declared non-significant. Asterisks indicate $\left(^{*}\right)$ significant correlation before adjustment for multiple testing and $\left(^{* *}\right)$ before and after adjustment for multiple testing.

MCP2 joint. Furthermore, similar results were found for osteitis, although correlation did not reach significance after adjustment. By contrast, there was no correlation between bone erosions and dGEMRIC suggesting that inflammation, but not bone erosion, is associated with cartilage microstructural changes in RA. T2 mapping showed an association with osteitis in most of the joint compartments with significance maintained after adjustments in the MCP2 joint (table 4 and online supplementary table). Thus, high values in T2 mapping, indicating more severe changes in cartilage microstructure, were related to a high degree of osteitis based on the RAMRIS scoring.

\section{DISCUSSION}

In this study, we have shown that biochemical properties of the articular cartilage of small finger joints of patients with established RA can be assessed in a suitable manner by high- resolution MRI using special sequences, such as dGEMRIC and T2 mapping. We could show that the biochemical composition of articular cartilage depends on the presence of macroanatomical alterations in the cartilage as well as the amount of synovitis and osteitis in the respective joint. Biochemical cartilage changes prevailed in the metacarpal heads as compared with the phalangeal bases, which could be based on either differences in the mechanical properties of these two compartments or differential exposure to synovial inflammatory tissue.

The fact that inflammatory features of RA, such as synovitis and osteitis, are strongly correlated with changes in cartilage microstructure is interesting. It supports the concept that synovitis and osteitis are the key triggers for direct damage of cartilage by producing matrix enzymes such as aggrecanases, which cleave the proteoglycans as well as matrix metalloproteinases, which digest the collagen fibres. ${ }^{20}$ It also may indicate that the actual inflammatory state of the joint is relevant for the cartilage properties supporting a dynamic time-dependent interaction between the amount of inflammatory tissue and cartilage function. At the moment, we do not know whether these biochemical changes of cartilage are reversible or not, however, they may do so in case of absent or only mild morphological changes. Thus, undulant burden of synovitis and osteitis in a joint may be flanked by reversible changes of cartilage biochemistry.

In support of such concept, bone erosions, which are clearly a cumulative indicator for past (and present) exposure of the joint to inflammation, were not related to cartilage microstructural changes as measured by dGEMRIC and T2 mapping. We did not find any correlation between the microanatomical cartilage changes and clinical as well as biochemical instruments for assessment of the global burden of inflammation, such as the DAS28 and the level of CRP, respectively. This latter finding may not be surprising, since all these analysis were based on the individual joint but not on systemic level, and is also in accordance with previous data from Miese and colleagues who have shown no correlation between dGEMRIC indices and clinical activity parameters of RA. ${ }^{7}$

In this study, we also differentiated between ICJS and IBJS, which is important, as the IBJS always represents the composite of ICJS and the CT. Consequently, radiographic joint space narrowing can result from changes in CT or joint space width. In support of the use of the IBJS, we found a very strong correlation between IBJS and ICJS. Moreover, the IBJS was also highly correlated with CT suggesting this instrument potentially being a very good surrogate for cartilage changes in RA. These data extend recent observations by Peterfy and colleagues showing that conventional $1.5 \mathrm{~T}$ MRI has high specificity and accuracy in depicting joint space narrowing when compared with conventional radiography. ${ }^{21}$ Similar results were also shown by the group of McQueen assessing the wrist joints of patients with RA $^{22}$ and by the OMERACT MRI Task Force. ${ }^{23}$ We also found a strong correlation between these macroscopic values of cartilage morphology and microstructural changes. Indeed, this finding is not surprising, as it suggests that in case of advanced cartilage damage, also microstructural parameters are altered.

The strength of the study includes the very rigorous assessment of all macroanatomical and microanatomical structures of the joint including the synovial membrane, the bone marrow and the bone itself by high-end imaging. Also, we assessed several macroanatomical parameters of cartilage, such as ICJS and IBJS, as well the CT in six separate areas. Moreover, microanatomical changes were assessed repeatedly, and by two 
independent readers in altogether 12 different regions of the two joints. We also performed a very stringent data analysis and also did not exclude any 'outliers' in dGEMRIC and T2 results, as normal values are not yet defined. The lack of a longitudinal follow-up of the patients is a limitation of the study, although this study was not designed to address the changes of cartilage in a time-dependent manner, but was to relate biochemical cartilage properties to standard morphological MRI results in RA. Moreover, the presence of outliers in the values for the ICJS suggests that measurement of the ICJS is tricky based on the minuscule distances assessed. As a consequence, evaluation of the ICJS is challenging due to technical limitations. By contrast, standard RAMRIS parameters were highly consistent. A further limitation of the study is the small patient number; thus, further and independent studies are needed to confirm these results. Especially, the absolute values of dGEMRIC and T2 measurements vary among different studies; at the moment, there is no standard protocol for performing biochemical cartilage imaging in the small joints, which complicates the comparison of individual studies.

In summary, our data indicate that the fine biochemical and microanatomical changes in articular cartilage in patients with RA are strongly related to the actual burden of inflammation, in particular osteitis and synovitis, but not to bone erosion. These findings shed new light on the interaction between inflammation and joint destruction in RA.

Acknowledgements This study was supported by the Deutsche Forschungsgemeinschaft (FG 661/TP4 and SPP1468-IMMUNOBONE), the Bundesministerium für Bildung und Forschung (BMBF; project ANCYLOSS), the MASTERSWITCH, OSTEOIMMUNE and TEAM projects of the European Union, as well as the IMI funded project BTCure.

Contributors $B H, A A, N R, P S, K E$ and RJ collected the data. ME, GHW, MU, DP and LL designed the study. BH, GS and SF wrote the manuscript.

Funding Deutsche Forschungsgemeinschaft; Bundesministerium für Bildung und Forschung; European Union; IMI.

Competing interests None.

Provenance and peer review Not commissioned; externally peer reviewed.

\section{REFERENCES}

1 Larsen A, Edgren J, Harju E, et al. Interobserver variation in the evaluation of radiologic changes of rheumatoid arthritis. Scand J Rheumatol 1979;8:109-12.

2 Sharp JT, Young DY, Bluhm GB, et al. How many joints in the hands and wrists should be included in a score of radiologic abnormalities used to assess rheumatoid arthritis? Arthritis Rheum 1985;28:1326-35.

3 Sharp JT, Wolfe F, Mitchell DM, et al. The progression of erosion and joint space narrowing scores in rheumatoid arthritis during the first twenty-five years of disease. Arthritis Rheum 1991;34:660-8.

4 van der Heijde DM, van Leeuwen MA, van Riel PL, et al. Radiographic progression on radiographs of hands and feet during the first 3 years of rheumatoid arthritis measured according to Sharp's method (van der Heijde modification). I Rheumatol 1995;22:1792-6.
5 Aletaha D, Funovits J, Smolen JS. Physical disability in rheumatoid arthritis is associated with cartilage damage rather than bone destruction. Ann Rheum Dis 2011;70:733-9.

6 Smolen JS, van der Heijde DM, Keystone EC, et al. Association of joint space narrowing with impairment of physical function and work ability in patients with early rheumatoid arthritis: protection beyond disease control by adalimumab plus methotrexate. Ann Rheum Dis 2013;72:1156-62.

7 Miese F, Buchbender C, Scherer A, et al. Molecular imaging of cartilage damage of finger joints in early rheumatoid arthritis with delayed gadolinium-enhanced magnetic resonance imaging. Arthritis Rheum 2012;64:394-9.

8 Bashir A, Gray ML, Burstein D. Gd-DTPA2- as a measure of cartilage degradation. Magn Reson Med 1996;36:665-73.

9 Andreisek G, White LM, Yang Y, et al. Delayed gadolinium-enhanced MR imaging of articular cartilage: three-dimensional T1 mapping with variable flip angles and B1 correction. Radiology 2009;252:865-73.

10 Trattnig S, Marlovits S, Gebetsroither S, et al. Three-dimensional delayed gadolinium-enhanced MRI of cartilage (dGEMRIC) for in vivo evaluation of reparative cartilage after matrix-associated autologous chondrocyte transplantation at 3.0T: preliminary results. JMRI 2007;26:974-82.

11 Tiderius CJ, Sandin J, Svensson J, et al. Knee cartilage quality assessed with dGEMRIC in rheumatoid arthritis patients before and after treatment with a TNF inhibitor. Acta Radiol 2010;51:1034-7.

12 van Tiel J, Bron EE, Tiderius CJ, et al. Reproducibility of 3D delayed gadolinium enhanced MRI of cartilage (dGEMRIC) of the knee at $3.0 \mathrm{~T}$ in patients with early stage osteoarthritis. Eur Radiol 2013;23:496-504.

13 Apprich S, Welsch GH, Mamisch TC, et al. Detection of degenerative cartilage disease: comparison of high-resolution morphological MR and quantitative T2 mapping at 3.0 Tesla. Osteoarthritis Cartilage 2010;18:1211-17.

14 Niemien MT, Rieppo J, Toyras J, et al. T2 relaxation reveals spatial collagen architecture in articular cartilage: a comparative quantitative MRI and polarized light microscopic study. Magn Reson Med 2001;46:487-93.

15 Miese FR, Ostendorf B, Wittsack HJ, et al. Metacarpophalangeal joints in rheumatoid arthritis: delayed gadolinium-enhanced MR imaging of cartilage-a feasibility study. Radiology 2010;257:441-7.

16 Buchbender C, Scherer A, Kropil P, et al. Cartilage quality in rheumatoid arthritis: comparison of T2* mapping, native T1 mapping, dGEMRIC, DeltaR1 and value of pre-contrast imaging. Skel Radiol 2012;41:685-92.

17 Ostergaard M, Edmonds J, McQueen F, et al. An introduction to the EULAR-OMERACT rheumatoid arthritis MRI reference image atlas. Ann Rheum Dis 2005;64(Suppl 1):i3-7.

18 Conaghan P, Bird P, Ejberg B, et al. The EULAR-OMERACT rheumatoid arthritis MRI reference image atlas: the metacarpophalangeal joints. Ann Rheum Dis 2005;64 (Suppl 1):i11-21.

19 Aletaha D, Neogi T, Silman AJ, et al. 2010 Rheumatoid arthritis classification criteria: an American College of Rheumatology/European League Against Rheumatism collaborative initiative. Arthritis Rheum 2010;62:2569-81.

20 Mclnnes IB, Schett $G$. Cytokines in the pathogenesis of rheumatoid arthritis. Nat Rev Immunol 2007;7:429-42.

21 Peterfy CG, Dicarlo JC, Olech E, et al. Evaluating joint-space narrowing and cartilage loss in rheumatoid arthritis by using MRI. Arthritis Res Ther 2012;14: R131.

22 McQueen F, Clarke A, McHaffie A, et al. Assessment of cartilage loss at the wrist in rheumatoid arthritis using a new MRI scoring system. Ann Rheum Dis 2010;69:1971-5.

23 Ostergaard M, Bøyesen P, Eshed I, et al. Development and preliminary validation of a magnetic resonance imaging joint space narrowing score for use in rheumatoid arthritis: potential adjunct to the OMERACT RA MRI scoring system. J Rheumatol 2011;38:2045-50. 


\title{
Osteitis and synovitis, but not bone erosion, is associated with proteoglycan loss and microstructure damage in the cartilage of patients with rheumatoid arthritis
}

Barbara Herz, Andreas Albrecht, Matthias Englbrecht, Götz H Welsch, Michael Uder, Nina Renner, Philipp Schlechtweg, Dominik Paul, Lars Lauer, Klaus Engelke, Rolf Janka, Jürgen Rech, Georg Schett and Stephanie Finzel

Ann Rheum Dis 2014 73: 1101-1106 originally published online April 26, 2013

doi: 10.1136/annrheumdis-2012-202850

Updated information and services can be found at:

http://ard.bmj.com/content/73/6/1101

Supplementary
Material

References

Email alerting service
These include:

Supplementary material can be found at:

http://ard.bmj.com/content/suppl/2013/04/25/annrheumdis-2012-2028 50.DC1.html

This article cites 21 articles, 6 of which you can access for free at: http://ard.bmj.com/content/73/6/1101\#BIBL

Receive free email alerts when new articles cite this article. Sign up in the box at the top right corner of the online article.

\section{Topic \\ Collections}

Articles on similar topics can be found in the following collections

\author{
Degenerative joint disease (4622) \\ Musculoskeletal syndromes (4931) \\ Bone and joint infections (66) \\ Calcium and bone (722) \\ Connective tissue disease (4235) \\ Immunology (including allergy) (5119) \\ Rheumatoid arthritis (3245) \\ Clinical diagnostic tests (1279) \\ Radiology (1112) \\ Radiology (diagnostics) (750)
}

\section{Notes}

To request permissions go to:

http://group.bmj.com/group/rights-licensing/permissions

To order reprints go to:

http://journals.bmj.com/cgi/reprintform

To subscribe to BMJ go to:

http://group.bmj.com/subscribe/ 